\title{
Treatment of Chronic Lateral Epicondylitis: Platelet Rich Plasma versus Extra-Corporeal Shock Wave Therapy
}

\author{
Ahmed Mohamed Ahmed Othman \\ Assistant Professor of Orthopedics, El-Minia University, El-Minia, Egypt \\ Email: osman682012@yahoo.com \\ Received 13 January 2014; revised 17 February 2014; accepted 26 February 2014 \\ Copyright (C) 2014 by author and Scientific Research Publishing Inc. \\ This work is licensed under the Creative Commons Attribution International License (CC BY). \\ http://creativecommons.org/licenses/by/4.0/

\section{Open Access}

\begin{abstract}
Background: Several treatment options have been proposed to treat lateral epicondylitis. Both Platelet rich plasma (PRP) and Extra-corporeal Shock Wave Therapy (ESWT) are new treatment modalities for tendinopathy. Patients and Methods: Thirty seven patients suffering from chronic lateral epicondylitis (tennis elbow) for at least six months were treated in this study. The patients were divided into two treatment groups, the first group including 20 patients treated by ESWT and the second group including 17 patients treated by local injection of platelet-rich plasma. The results were evaluated using the visual analogue scale and the DASH score. Results: In the first group (treated by ESWT), the average follow-up period was 21.55 months. The average VAS improved from 8.2 to 1.95 and the average DASH score improved from 72.25 to 51.7. Moreover, 11 cases were satisfied $(55 \%), 3$ cases $(15 \%)$ were satisfied with reservation and 6 cases $(30 \%)$ were not satisfied. In the second group (treated by PRP), the average follow-up period was 18.47 months. The average VAS improved from 8.52 to 1.47 , the DASH score improved from 72 to 48.23. Concerning patient satisfaction, 13 cases were satisfied $(83.33 \%), 3$ cases $(10.52 \%)$ were satisfied with reservation and 1 case (5.55\%) was not satisfied. Conclusion: Platelet rich plasma was proved to achieve superior results when compared to ESWT as regards pain relief, improvement of elbow function and patient satisfaction at follow-up.
\end{abstract}

\section{Keywords}

Lateral Epicondylitis; ESWT; PRP; DASH Score

\section{Introduction}

Tennis elbow is the most common cause of elbow pain in patients attending the orthopedic clinics. It occurs 
most commonly in the tendon of extensor carpi radialis brevis [1].

However, other tendons of the extensor bundle such as the extensor digitorum communis (involved in $30 \%$ of cases) may be involved [2].

Pathophysiology involves microtears in the tendon leading to hemorrhage, rough granulation tissue formation and later repair [3].

The initial management of tennis elbow is conservative, with the use of rest, activity modification, non-steroidal anti-inflammatory drugs, forearm bracing, physiotherapy and local steroid injections. These measures result in up to $90 \%$ of patients being in a transient remission [4] [5].

Even though the different treatment modalities of tennis elbow have been claimed to be effective in treating this condition due to its chronic nature and tendency to recur with resumption of activity, no single modality has been considered [1].

Newer treatment modalities have been tried such as extracorporeal shock wave therapy, iontophoresis and injection of botulism toxin. If conservative treatment fails, the last resort is surgery with the primary objective of pain relief [6]-[8].

Shock wave therapy has been employed in orthopedic and traumatic conditions including calcific tendonitis, tennis elbow, plantar fasciitis, achilles tendonitis, patellar tendonitis fracture non-unions and pseudoarthrosis as well as myositis ossificans and avascular necrosis [9].

The mechanism of action of ESWT is unknown, but it possibly includes direct stimulation of the healing process, neovascularization, disintegration of calcium and neural elements. These may involve alterations of the permeability of cell membranes preventing the development of potentials to transmit painful stimuli, direct suppression on nociceptors and a hyperstimulation mechanism which controls gate control mechanism [10] [11].

Growth factor technologies are increasingly used to enhance healing in musculoskeletal injuries, particularly in sports medicine [12].

Platelet rich plasma contains more concentrated amounts of platelets than does whole blood. Within the platelets are powerful growth factors, including platelet-derived growth factors, transforming growth factor beta, and epidermal growth factor [13].

Moreover, platelet rich plasma (PRP) has shown to be a general stimulator for repair or regeneration [14] [15].

Sonographic assessment proved that platelet rich plasma improves extensor tendon morphology after local injection [16].

Platelet-rich plasma has been shown to reduce pain and symptoms of lateral epicondylitis [2].

\section{Material and Methods: (Table 1)}

The study included two groups of patients according to the methods of treatment either by ESWT or PRP.

\subsection{Inclusion Criteria}

1) Pain over the common extensor origin increases with pressure over the lateral epicondyle and with resisted dorsiflexion of the wrist and or middle finger.

2) Duration of symptoms for at least 6 months.

3) Pain ranking at least 5 on a 10 -point scale.

Table 1. Pre-treatment data of both groups.

\begin{tabular}{cccc}
\hline & ESWT & PRP & \\
Age (years) & $26-53(37.3)$ & $23-57(36.76)$ & $13 / 4$ \\
Sex (Male/Female) & $15 / 5$ & 6.807007 \\
Pre-treatment period (months) & $6-36(17.95)$ & $7-9(8.52)$ & 0.853325 \\
Pre-treatment VAS & $6-9(8.2)$ & $60-88(72)$ & 0.110115 \\
Pre-treatment DASH score & $60-88(72.25)$ & 0.306979 \\
\hline
\end{tabular}




\subsection{Exclusion Criteria}

1) Active inflammatory disease.

2) Concurrent pain in the cervical spine or ipsilateral shoulder due to other cause.

3) Coagulation disorders.

4) Previous treatment with steroids for the last 3 months.

The method of treatment was discussed with the patient before the start of treatment. The ESWT technique depends upon application of 3 sessions of ESWT without anesthesia with one week interval between each session whereas PRP method depend withdrawal of patient's own blood, centrifugation and injection into the affected tendon. So, the choice of the method of treatment was based on patient's choice after discussing the two techniques with the patient.

The first group included 20 patients treated by ESWT 15 males and 5 females, all cases were unilateral, the age of the patients ranged between 26 to 53 years with an average of 37.3 years. The pre-treatment period ranged between 6 months and 35 months with an average of 17.95 months. The pre-treatment VAS scale ranged between 6 to 9 points with an average of 8.2 and the pre-treatment DASH score ranged between 60 to 88 with an average of 72.25 .

The second group included 17 patients treated by PRP injection 13 males and 4 females, all cases were unilateral, the age of the patients ranged between 23 to 57 years with an average of 36.76 years. The pre-treatment period ranged between 6 months and 36 months with an average of 17.88 months. The patients were evaluated pre-operatively using the Visual Analogue Scale (VAS) and the Disabilities of the Arm, Shoulder and Hand (DASH) score. The pre-treatment VAS scale ranged between 7 to 9 points with an average of 8.52 and the pretreatment DASH score ranged between 60 to 88 with an average of 73.

The patient is seen after treatment in the first day, after 1 week, one month and then every three months for evaluation of pain and elbow function.

\subsection{Technique of ESWT}

Every patient is given three sessions of ESWT at weekly intervals. A total of 2000 shock waves (at 0.07 to 0.09 $\mathrm{mJ} / \mathrm{mm}^{2}$ ) were administered at each session under ultrasound guide and without anesthesia.

\subsection{Technique of PRP}

The procedure is performed in the outpatient surgery centre.

1) First, between 45 - $60 \mathrm{ml}$ of patients own blood is withdrawn.

2) This amount is centrifuged and the platelet rich plasma portion is collected in a syringe.

3) While the centrifuge process is going on, local anesthetic is injected after skin preparation of the area of the tendon.

4) Then, the PRP is injected into the tendon using 22-g needle by peppering technique.

\subsection{Post-Injection Protocol}

The patient is kept in supine position without moving for 15 minutes. Patients are sent home with instructions to limit the use of the arm for 24 hours and given paracetamol or codeine for pain but not given NSAIDs.

After 24 hours, patients were given a standardized stretching protocol for 2 weeks. A formal strengthening program is then initiated. At 4 weeks after the procedure, patients were allowed to proceed with normal sporting or recreational activities as tolerated.

\section{Statistical Analysis}

The SPSS (version 11.5, SPSS Inc, Chicago, IL, USA) was used. Chi square test were used to compare the results between the two groups. The Kruskal-Wallis test was applied to compare VAS score changes before and after treatment. The difference in the VAS at the early results and at follow up was estimated using the Wilcoxon signed ranks test. Concerning the demographic data including the age $(P=0.807007)$, pre-treatment period $(P=0.853325)$, VAS $(P=0.110115)$ and DASH score $(P=0.30697)$, consequently, there was no significant difference. 


\section{Results: (Tables 2-4)}

In the first group (treated by ESWT), all cases were unilateral and all patients were available for follow up. The follow up period ranged between 8 months and 40 months with an average of 21.55 months. The average VAS improved from 8.2 to 1.95 with a mean improvement of $6.25(P=0.0001)$ and the average DASH score improved from 72.25 to 51.7 with a mean improvement of $20.55(P=0.0001)$. Concerning patient satisfaction, 11 cases were satisfied (55\%), 3 cases (15\%) were satisfied with reservation and 6 cases (30\%) were not satisfied. The success rate was $70 \%$.

In the second group (treated by PRP), all cases were unilateral, The follow up period ranged between 7 months and 33 months with an average of 18.47 months. The average VAS improved from 8.52 to 1.47 with a mean improvement of $7.05(P=0.0001)$, the DASH score improved from 72 to 48.23 with a mean improvement of $23.77(P=0.0001)$. Concerning patient satisfaction, 13 cases were satisfied (83.33\%), 3 cases $(10.52 \%)$ were satisfied with reservation and 1 case $(5.55 \%)$ was not satisfied. The success rate was $93.85 \%$.

There were no reported complications in either group either early or at late follow up.

In the post-treatment results of the ESWT group, the mean change in the VAS was $6.25(P=0.0001)$ and the mean change in the DASH score was $20.55(P=0.0001)$. In the PRP group, the mean change in the VAS was $7.05(P=0.001)$ and the mean change in the DASH score was $23.77(P=0.001)$.

Regarding the difference in the results between the two groups, the mean improvement in the in the VAS in the ESWT group was 6.25 and in the PRP group was $7.05(P$ value $=0.05)$ and in the DASH score, the mean improvement in the ESWT group was 20.55 and in the PRP group was 23.77 ( $P$ value $=0.05$ ). Accordingly, there was a significant difference in the results of both groups in favor of the PRP group.

Outcome and the success rate, the PRP group achieved much better results than the ESWT group i.e. 93.85\% versus $70 \%$ respectively $(P=0.001)$.

Successful results were defined as reduction of as much as $25 \%$ of VAS and DASH score in either group at late follow up. Consequently, the PRP group achieved more success as compared to ESWT group $(P<0.001)$.

\section{Discussion}

Elbow epicondylar tendinosis is a common problem with many available treatments. Quick cessation of symptoms is important and is economically advantageous. If neither rest nor simple treatment provides a satisfactory

Table 2. Post-treatment results of the whole study.

\begin{tabular}{cccc}
\hline & ESWT & PRP & $P$ value \\
\hline Average follow up period (months) & 21.55 & 18.47 & 0.376395 \\
Average Post-treatment VAS & $1-4(1.95)$ & $1-3(1.47)$ & 0.02 \\
Average Post-treatment DASH score & $46-58(51.7)$ & $40-54(48.23)$ & 0.01 \\
\hline
\end{tabular}

Table 3. Results of the whole study.

\begin{tabular}{ccc}
\hline & ESWT & PRP \\
\hline Satisfied & $11(55 \%)$ & $3.33 \%)$ \\
Satisfied with reservation & $3(15 \%)$ & $(10.52 \%)$ \\
Not satisfied & $6(30 \%)$ & $1(5.5 \%)$ \\
\hline
\end{tabular}

Table 4. Mean changes in DASH score and VAS.

\begin{tabular}{ccc}
\hline & ESWT & PRP value \\
\hline Mean improvement in VAS & 6.25 & 7.05 \\
Mean improvement in VAS DASH score & 20.55 & 23.77 \\
\hline
\end{tabular}


remedy, a patient may pursue other treatment options [13].

Non-operative management will result in successful resolution of symptoms in $90 \%$ of patients [2].

Rompe et al., (1996) [17] have described the use of low-dose ESWT in patients with chronic tennis elbow. There was a significant reduction in pain and increase in grip strength in the group which received shock wave therapy (3000 impulses of $0.08 \mathrm{MJ} / \mathrm{mm}^{2}$ ).

Haake et al., (2002) [18] in a double blind controlled study involved 270 patients with tennis elbow received either ESWT (2000 shocks at 0.07 to $0.09 \mathrm{~mJ} / \mathrm{mm}^{2}$ ) or placebo at weekly intervals for three weeks, no difference was noted between the two groups.

Pettrone and McCall (2005) [19] found significant improvement after full dose of ESWT at 3 months ranged between $33 \%$ to $70 \%$ and at 6 months ranged between $55 \%$ to $79 \%$.

Staples et al., (2008) [20] conducted a double-blind randomized controlled trial on 68 patients to determine whether ultrasound-guided ESWT reduced pain and improved function in patients with lateral epicondylitis. Patients received 3 ESWT treatments or 3 treatments at a subtherapeutic dose given at weekly intervals. There were significant improvements in almost all outcome measures for both groups over the 6 months follow up. There was little evidence to support the use of ESWT at a therapeutic or subtherapeutic dose for the treatment of lateral epicondylitis.

Many articles have studied the use of PRP in treatment of lateral epicondylitis in the past few years.

Mishra and Pavelko (2006) [13] treated 140 patients with chronic lateral epicondylitis by injection of plateletrich plasma and at final follow up (mean 25.6 months; range 12 - 38 months) they reported 93\% reduction in pain compared with before treatment.

Hechtman et al., (2011) [21] in a similar study using PRP, treated 31 patients with epicondylitis not responding to conservative treatment for 6 months. Two cases elected surgery 1 month post-injection and 29 cases continued follow up. The overall success rate was $90 \%$ (28 of 31 elbows). Patient satisfaction improved from $5.1 \pm$ 2.5 at 1 month to $9.1 \pm 1.9$ at last follow up.

Peerbooms et al., (2010) [14] compared the results of two groups of patients suffering from lateral epicondylitis. The first group $(n=51)$ treated by PRP injection and the second group $(n=49)$ treated by corticosteroid injection. The results showed $73 \%$ success rate in the PRP group compared to $51 \%$ success rate in the corticosteroid group after 1 year follow up.

Thanasas et al. (2011) [22] treated two groups of patients with lateral epicondylitis the first group included 14 patients treated by PRP injection and the second group included 14 patients treated by injection of autologous blood and concluded that PRP treatment is superior to autologous blood concerning short term results but no statistically significant difference in the elbow function at follow up.

Creaney et al., (2011) [12] compared the results of two groups of patients suffering from lateral epicondylitis. The first group $(n=80)$ treated by PRP injection and the second group $(n=70)$ treated by autologous blood injection. The results showed $66 \%$ success rate in the PRP group compared to $72 \%$ success rate in the autologous blood injection group after 6 months follow up.

Gosens et al., (2011) [23] compared the results of two groups of patients with chronic lateral epicondylitis. The first group $(n=51)$ treated by leukocyte-enriched PRP injection and the second group $(n=49)$ treated by corticosteroid injection. Both groups significantly improved across the time. After 2 years follow up, the DASH scores of the corticosteroid group returned to baseline levels while those of PRP group significantly improved.

Chaudhury et al., (2012) [16] treated 6 patients who had a baseline ultrasound confirming tendinosus of the common extensor tendon. Patients received $3 \mathrm{ml}$ PRP under sonographic control. Five patients were available for follow up and they gained improvement of tendon morphology rather than tendon vascularity at 6 months follow up.

We did not find in the literature any available study comparing the results of ESWT with those of PRP in treating lateral epicondylitis. In the current study, the results showed that both ESWT and PRP can be used for treatment of such a condition. However, PRP group of patients achieved better results and more patient satisfaction. In the PRP group, the overall success rate was 93.85\% (16 of 17 elbows) whereas in the ESWT group it was $80 \%$ (16 out of 20 elbows). The recorded levels of patient satisfaction were much higher in the PRP group. The assessment of our results was based totally on the subjective and clinical data during the pre-treatment and post-treatment periods. May be it will be more reliable to use ultrasonographic measurement of the thickness and vascularity of the common extensor tendon at the start of treatment and at late follow up. 


\section{Conclusion}

Lateral epicondylitis or tennis elbow can be treated effectively with non-surgical methods of treatment. Both ESWT and PRP can be successfully used for treatment of tennis elbow. However, PRP is more effective concerning pain relief and improvement of elbow function. PRP is a promising technique for treatment of tendinopathy.

\section{Strengths and Limitations of This Work}

The proposed techniques provide a new scale of conservative treatment for chronic tennis elbow without the need for surgery and with promising early results. The limitations in our study were small size of the study groups as well as short period of follow up, and longer periods of follow up as well as larger sizes of study are need.

\section{References}

[1] Nirschel, R.P. and Pettrone, F.A. (1997) The Surgical Treatment of Lateral Epicondylitis. The Journal of Bone \& Joint Surgery, 61, 832-839.

[2] Taylor, S.A. and Hannafin, J.A. (2012) Evaluation and Management of Elbow Tendinopathy. Sports Health: A Multidisciplinary Approach, 4, 384.

[3] Vicenzino, B. and Wright, A. (1996) Lateral Epicondylagia. Epidemiology, Pathophysiology, Etiology and Natural History. Physical Therapy Reviews, 1, 23-34. http://dx.doi.org/10.1179/ptr.1996.1.1.23

[4] Coonrad, R.W. and Hooper, W.R. (1973) Tennis Elbow: Its Natural Course, Natural History, Conservative and Surgical Management. JBJS, 55A, 1177-1182.

[5] Price, R., Sinclair, H., Heinrich, I. and Gibson, T. (1991) Local Injection Treatment of Tennis Elbow; Hydrocortisone, Triamnicinolone and Lidocaine Compared. British Journal of Rheumatology, 30, 39-44. http://dx.doi.org/10.1093/rheumatology/30.1.39

[6] Andres, B.M. and Murrel, G.A. (2008) Treatment of Tendinopathy: What Works, What Doesn'T and Is on the Horizon. Clinical Orthopaedics and Related Research, 466, 1539-1554.

[7] Alsousou, J., Thompson, M., Hulley, P., Nobel, A. and Wlett, K. (2009) The Biology of Platelet-Rich Plasma and Its Application in Trauma and Orthopedic Surgery: A Review of the Literature. The Journal of Bone \& Joint Surgery, Br., 91, 987-996. http://dx.doi.org/10.1302/0301-620X.91B8.22546

[8] Scher, D.L., Wolf, J.M. and Owens, B.D. (2009) Lateral Epicondylitis. Orthopedics, 32, 276-281. http://dx.doi.org/10.3928/01477447-20090401-06

[9] Haupt, G. (1997) Review Article: Use of Extracorporeal Shock Waves in the Treatment of Pseudoarthrosis, Tendinopathy, and Other Therapeutic Cases. Journal of Urology, 158, 4-11. http://dx.doi.org/10.1097/00005392-199707000-00003

[10] Schelling, G., Delius, M., Gscwender, M., Grafe, P. and Gambihler, S. (1994) Extra-Corporeal Shock Waves Stimulate Frog Sciatic Nerves Indirectly via a Cavitation Mediated Mechanism. Biophysics, 66, 133-140.

[11] Wang, C., Huang, H.Y. and Pai, C.H. (2002) Shock Wave-Enhanced Neovascularization at the Tendon Bone Junction: An Experiment in Dogs. Journal of Foot and Ankle Surgery, 41, 16-22. http://dx.doi.org/10.1016/S1067-2516(02)80005-9

[12] Creaney, L., Wallace, A., Curtis, M and Conell, D. (2011) Growth Factor-Based Therapies Provide Additional Benefit beyond Physical Therapy in Resistant Elbow Tendinopathy: A Prospective, Single Blind Randomized Trial of Autologous Blood Injections versus Platelet-Rich Plasma Injections. British Journal of Sports Medicine, 45, 966-971. http://dx.doi.org/10.1136/bjsm.2010.082503

[13] Mishra, A. and Pavelko, T. (2006) Treatment of Chronic Elbow Tendinosis with Buffered Platelet-Rich Plasma. American Journal of Sports Medicine, 34, 17774-17778. http://dx.doi.org/10.1177/0363546506288850

[14] Peerbooms, J.C., Sluimer, J., Bruijn, D.J. and Gosens, T. (2010) Positive Effect of an Autologous Platelet Concentrate in Lateral Epicondylitis in a Double-Blind Randomized Controlled Trial: Platelet-Rich Plasma versus Corticosteroid Injection with a 1year Follow-Up. American Journal of Sports Medicine, 38, 255-262. http://dx.doi.org/10.1177/0363546509355445

[15] Mishra, A., Randelli, P., Barr, C., Talamonti, T., Raone, V. and Cabitza, P. (2012) Platelet-Rich Plasma and the Upper Extremity. Hand Clinics, 28, 481-491. http://dx.doi.org/10.1016/j.hcl.2012.08.008

[16] Chaudhury, S., de lama, M., Alder, R.S., Gultta, L.V., Skonieczki, B., Moley, P., Cordasco, E., Hannafin, J. and Fealy, 
S. (2012) Platelet-Rich Plasma and the Upper Extremity. Skeletal Radiology, [Epub ahead of print].

[17] Rompe, J.D., Hopf, C., Kullmer, K., Heine, J. and Burger, R. (1996) Analgesic Effect of Extracorporeal Shock Wave Therapy on Chronic Tennis Elbow. The Journal of Bone \& Joint Surgery, 78, 233-237.

[18] Haake, M., Koni, I.R. and Decker, T. (2002) No Effectiveness of Extracorporeal Shock Wave Therapy in the Treatment of Tennis Elbow: Results from Prospective Randomized Placebo-Controlled Multicenter Trial. The Journal of Bone \& Joint Surgery, 84-A, 1982-1991.

[19] Pettrone, F.A. and McCall, B.R. (2005) Extracorporeal Shock Wave Therapy without Local Anesthesia for Chronic Lateral Epicondylitis. The Journal of Bone \& Joint Surgery, 87-A, 1297-1304. http://dx.doi.org/10.2106/JBJS.C.01356

[20] Staples, M.P., Forbes, A. and Patznik, R. (2008) A Randomized Controlled Trial of Extracorporeal Shock Wave Therapy for Lateral Epicondylitis. Journal of Rheumatology, 35, 2038-2046.

[21] Hechtman, S., Uribe, J., Botto-van Demden, A. and Kiebzak, G.M. (2011) Platelet-Rich Plasma Injection Reduces Pain in Patients with Recalcitrant Epicondylitis. Orthopedics, 34, 2-92.

[22] Thanasas, C., Papadimtriou, G., Charalambidis, C., Paraskevopouols, I. and Papanikolau, A. (2011) Platelet Rich Plasma versus Autologous Blood for the Treatment of Chronic Lateral Elbow Epicondylitis: A Randomized Controlled Trial. American Journal of Sports Medicine, 39, 2134-2134. http://dx.doi.org/10.1177/0363546511417113

[23] Gosens, T., Peerbooms, J.C., van Laar, W. and den Oudsten, B.L. (2011) Ongoing Positive Effect of Platelet Rich Plasma versus Corticosteroid Injection in Lateral Epicondylitis: A Double-Blind Randomized Controlled Trial: Platelet-Rich Plasma versus with 2 Years Follow-Up. American Journal of Sports Medicine, 39, 1200-1208. http://dx.doi.org/10.1177/0363546510397173

\section{List of Abbreviations}

ESWT: Extra Corporeal Shock Wave Therapy;

PRP: Platelet Rich Plasma;

VAS: Visual Analogue Scale;

DASH Score: The Disabilities of the Arm, Shoulder and Hand (DASH) Score. 